\title{
The Effects of Dexmedetomidine on Airway-Related Complications During Emergence from General Anesthesia in Nasal and Paranasal Surgeries
}

\section{Nabeel Ibrahim Fathy Elmasry, Maamoun Mohamed Ismael, Amr Soliman Hamroush, Ahmed Gamal} Moustafa Hegazy*

Anesthesiology and Intensive Care Department, Faculty of Medicine, Al-Azhar University

* Corresponding author: Ahmed Gamal Moustafa Hegazy, E-mail: ahmedhegazy651 @ yahoo.com

\section{ABSTRACT}

Background: Stimulation of various sites, from the nasal mucosa to the diaphragm, can evoke laryngospasm. To reduce airway reflexes, tracheal extubation should be performed by special technique or with drugs that do not depress ventilation. However, tracheal extubation during rhinoplasty may be difficult because of the aspiration of blood and the possibility of laryngospasm. Dexmedetomidine has sedative and analgesic effects, without affecting respiratory status.

Objectives: To evaluate the effects of dexmedetomidine on airway-related complications during emergence from general anesthesia (GA) in nasal and paranasal surgeries.

Patients and Methods: This prospective randomized clinical study was included 90 patients of both sexes, admitted for elective nasal and paranasal sinus surgery. They were randomly allocated into three groups 30 patients each, Group A: Standard awake extubation technique. Group B: fully awake "no stimulation extubation" technique. Group C: Dexmedetomidine-group, who received intravenous (I.V) dexmedetomidine $0.5-1 \mu \mathrm{g} / \mathrm{kg}$ bolus in $100 \mathrm{ml}$ of normal saline over 10 minutes before the end of surgery by 10 minutes, The dexmedetomidine bolus was followed by $0.2 \mu \mathrm{g} / \mathrm{kg} / \mathrm{hr}$ which was stopped immediately when extubation was done.

Results: The following parameters were assessed between the three groups: hemodynamics, airway-related complications, extubation time. Group C: was associated with a significant increase in extubation quality compared with group A and group B regarding hemodynamics, airway-related complications, extubation time.

Conclusion: This study showed that the dexmedetomidine group associated with minimal circulatory reflexes and airway-related complications further to the advantage of short extubation time compared with the "no stimulation" extubation technique.

Keywords: Airway, Awake, Complications, Emergence, Nasal sinus, surgery, Technique, Tracheal, "no stimulation" dexmedetomidine, Tracheal extubation, Rhinoplasty.

\section{INTRODUCTION}

Intubation and extubation are associated with cardiovascular and various airway responses leading to tachycardia, hypertension, arrhythmia, myocardial ischemia, cough induction, bronchospasm, increased bleeding, increased intracranial and intraocular pressure $^{(\mathbf{1})}$.

Extubation under deep anesthesia decreases cardiovascular stimulation and reduces the incidence of coughing and straining on the tube. However, the incidence of respiratory complications has been found to be greater after extubation under deep anesthesia, regardless of the type of operation (2). Smooth extubation requires the absence of straining, movement, coughing, breath holding and laryngospasm ${ }^{(3)}$.

Many theories have been described for sudden increase in heart rate (HR) and blood pressure (BP) during extubation such as surge of catecholamines, airway irritation, intense pain following surgery, and emergence. Although these changes are transitory, it could be a major concern for an anesthesiologist ${ }^{(4)}$.

Much attention has been paid to attenuate these changes during extubation. Intratracheal LA instillation, ${ }^{(5)}$ I.V lignocaine, ${ }^{(6)}$ short acting opioids such as fentanyl and remifentanil, ${ }^{(7)}$ esmolol, ${ }^{(8)}$ labetalol, ${ }^{(\mathbf{3})}$ diltiazem, ${ }^{(\mathbf{1 0})}$ and verapamil ${ }^{(\mathbf{1 1})}$ have been used to attenuate these hemodynamic and respiratory responses during extubation in the past but with certain limitations.

The "no stimulation" extubation technique has been suggested and avoids extubation under light plan of anesthesia and ensures extubation only when consciousness is returned. It requires absolutely no stimulation during emergence and performing extubation only when the patient wakes up spontaneously and opens his eyes ${ }^{\mathbf{( 1 2})}$.

Recently dexmedetomidine, a potent $\alpha 2$ adrenoreceptor agonist has been used to facilitate extubation in surgical intensive care unit, but its role in the attenuation of hemodynamic and airway reflexes during extubation in GA is still scarce. The effects of single dose of dexmedetomidine in attenuation of the airway and circulatory reflexes during extubation still under research ${ }^{(13)}$.

\section{AIM OF THIS STUDY}

The aim of the work was to evaluate the effects of single dose of dexmedetomidine on circulatory reflexes and airway-related complications during emergence from GA in nasal and paranasal surgeries. Hemodynamics, airway-related complications (coughing, bucking, laryngospasm, oozing from the wound, the incidence of vomiting), extubation time between three groups and determined convenient technique for tracheal extubation were investigated. 


\section{PATIENTS AND METHODS}

\section{Ethical approval:}

This prospective randomized single-blinded, comparative study was approved by the ethics committee in Al-Azhar University Hospitals (ElHussein and Sayed Galal) and patients' written informed consent was obtained.

Patients:

This study included 90 patients of both sexes, admitted for elective nasal and paranasal sinus surgery. They were randomly allocated into three equal groups, 30 patients each $(n=30)$ :

\section{technique}

Group (A): Standard awake extubation

Group (B): fully awake "no stimulation extubation" technique.

Group (C): Dexmedetomidine-group.

Inclusion criteria: patients with:

1- American Society of Anesthesiologist (ASA) class I and II.

2- Aged 18-35 years of both sexes.

3- Patients undergoing elective nasal and paranasal sinus surgery.

\section{Exclusion criteria:}

1- Patients under treatment with sedatives, antitussives, or angiotensin-converting enzyme inhibitors, history of current or chronic upper airway disease, asthma, chronic obstructive airway diseases and chronic cough.

2- Patients with systemic illnesses (eg, hypertension [systolic BP (SBP) >160 mm $\mathrm{Hg}$, diabetes mellitus.

\section{Preoperative evaluation:}

Routine preoperative assessment by full history taking, physical examination including chest and heart examination and investigations $(\mathrm{CBC}, \mathrm{S}$. creatinine, blood urea, AST, ALT, PT, PTT, INR, ECG, and chest X-Ray).

\section{Methods:}

All patients were premedicated with $10 \mathrm{mg}$ diazepam orally 2 hours before surgery, received 10$12 \mathrm{mg} / \mathrm{kg}$ of I.V paracetamol for postoperative analgesia, and I.V ondansetron $4 \mathrm{mg}$ for the prevention of postoperative nausea and vomiting thirty minutes before the end of surgery. The technique of GA was standardized for all patients where preoxygenation was done 3 minutes before induction of anesthesia, then all patients received fentanyl $1-2 \mu \mathrm{g} / \mathrm{kg}$ and after 2 minutes. Propofol was given in dose $2 \mathrm{mg} / \mathrm{kg}$ throughout 90 seconds and the endotracheal intubation facilitated with rocuronium $0.45-0.6 \mathrm{mg} / \mathrm{kg}$ IV. $1 \mu \mathrm{g} / \mathrm{kg}$ fentanyl was given to patient when signs of lack of analgesia recorded (MBP increase $20 \%$ of base line). the TT size was chosen 7 to $7.5 \mathrm{~mm}$ internal diameter in females and 8 to $8.5 \mathrm{~mm}$ internal diameter in males, and the cuff was inflated with air, and cuff-pressure was monitored and maintained at 20 mbar throughout the procedure. Topical nasal application of $1 \% \quad(10$ $\mathrm{mg} / \mathrm{ml}$ ) lidocaine with 1: 200000 adrenaline was used for all patients to reduce bleeding during surgery. Gauze throat pack was inserted under vision by direct laryngoscope in each patient and eye lubricant was applied.

After tracheal intubation, anesthesia was maintained with $2 \%$ sevoflurane in a mixture of $50 \%$ nitrous oxide in oxygen via a closed-circuit system. During mechanical ventilation the respiratory rate and tidal volume were adjusted to maintain the endtidal $\mathrm{CO} 2$ (ETCO $32-38 \mathrm{~mm} \mathrm{Hg}$ ) intraoperatively. Neuromuscular blockade was maintained with Rocuronium $0.3 \mathrm{mg} / \mathrm{kg}$ on demand. Basic monitoring for all patients consisted of noninvasive $\mathrm{BP}$ measurement, (HR), electrocardiograph, pulse oxygen saturation $(\mathrm{SpO} 2)$, end-tidal $\mathrm{CO} 2$ values and train-of-four monitoring.

Thirty minutes before the end of surgery, all patients received $10-12 \mathrm{mg} / \mathrm{kg}$ of I.V paracetamol for postoperative analgesia, and I.V ondansetron $4 \mathrm{mg}$ for the prevention of postoperative nausea and vomiting, during the last 20 minutes at the end of surgery any residual neuromuscular blockade, as determined by train-of-four monitoring, was reversed by I.V neostigmine $50 \mu \mathrm{g} / \mathrm{kg}$ (to a maximum of 5 $\mathrm{mg}$ ) plus atropine $10-20 \mu \mathrm{g} / \mathrm{kg}$. Nitrous oxide was discontinued, and the patient was allowed to breathe spontaneously sevoflurane and oxygen in air $40 \%$. At the end of surgery, the nose was packed by a nasal packing.

Before the end of surgery, all the ninety patients, were randomly allocated into one of three equal groups according to the method of extubation:

Group A: Standard awake extubation, after completion of the procedure, sevoflurane was immediately discontinued, and fresh gas flow was increased. Throat pack was removed, any blood and secretions in the pharynx were carefully suctioned, and the patients were then turned to the lateral (recovery) position. If the patient did not breath spontaneously, positive pressure ventilation was continued with $100 \%$ oxygen until spontaneous ventilation returned. Tracheal extubation was performed when the patient regained consciousness, had enough spontaneous breathing, intact gag reflex.

Group B: fully awake "no stimulation extubation" technique (absolutely no stimulation was allowed until patients were able to open their eyes), at the end of the procedure, while the patient was still adequately anesthetized, throat pack was removed, any blood and secretions in the pharynx were carefully suctioned under direct visualization in order to confirm that secretion clearance was complete and to avoid trauma to the mucosa. The TT cuff was 
deflated, and then the patients were turned to the lateral (recovery) position. After this, sevoflurane was discontinued and fresh gas flow was increased, and if the patient did not breath spontaneously, positive pressure ventilation was continued with $100 \%$ oxygen until spontaneous ventilation returned. Tracheal extubation was performed when patients had same criteria of group (A), but absolutely no stimulation was allowed until patients spontaneously woke up and were able to open their eyes. The anesthetist was only allowed to call their name or give a simple verbal command to open their eyes, without physically stimulating the patient, differently from group (A), in this technique, we avoided any stimulation such as oropharyngeal suctioning, head turning, pillow removal, and bodily movement, which all serve to reduce tracheal irritation from the endotracheal tube.

Group C: Dexmedetomidine - group, During the last 10 minutes before the end of surgery, after completion of the procedure, sevoflurane was immediately discontinued and fresh gas flow was increased, and started to give a bolus dose of dexmedetomidine $0.5-1 \mu \mathrm{g} / \mathrm{kg}$ in $100 \mathrm{ml}$ of normal saline over 10 minutes then, after bolus dose has been finished we started to give a continuous maintenance infusion dose of dexmedetomidine 0.2 $\mathrm{ug} / \mathrm{kg} / \mathrm{hr}$, which must be stopped when extubation was done. Throat pack was removed, any blood and secretions in the pharynx were carefully suctioned, and the patients were then turned to the lateral (recovery) position. If the patient did not breath spontaneously, positive pressure ventilation was continued with $100 \%$ oxygen until spontaneous ventilation returned. Tracheal extubation was performed when the patient regained consciousness, had sufficient spontaneous breathing, intact gag reflex, purposeful movement, and spontaneous eye opening.

After extubation in the three groups, the patients breathed $40 \%$ oxygen by face mask until they were able to maintain a patent airway, and consequently they were transported to the post anesthesia care unit (PACU). Pulse oximetry was provided continuously, intraoperatively, during transport, and throughout recovery period (was mandatory).

\section{Statistical analysis}

Recorded data were analyzed using the statistical package for social sciences, version 20.0 (SPSS Inc., Chicago, Illinois, USA). Quantitative data were expressed as mean \pm standard deviation (SD). Qualitative data were expressed as frequency and percentage. Probability (P-value): P-value $<0.05$ was considered significant, P-value $<0.001$ was considered as highly significant, $\mathrm{P}$-value $>0.05$ was considered insignificant.

\section{RESULTS}

Table (1): Comparison between the groups regarding demographic data.

\begin{tabular}{|c|c|c|c|c|c|}
\hline $\begin{array}{l}\text { Demographic } \\
\text { Data }\end{array}$ & $\begin{array}{c}\text { Group (A): } \\
\text { Standard } \\
(\mathrm{n}=\mathbf{3 0})\end{array}$ & $\begin{array}{c}\text { Group (B): No } \\
\text { stimulation } \\
(\mathbf{n}=\mathbf{3 0})\end{array}$ & $\begin{array}{c}\text { Group }(\mathrm{C}): \\
\begin{array}{c}\text { Dexmedetomidine } \\
(\mathrm{n}=30)\end{array}\end{array}$ & F/x2\# & p-value \\
\hline Age (years) & $27.29 \pm 5.18$ & $28.36 \pm 5.39$ & $28.89 \pm 5.49$ & 0.784 & 0.436 \\
\hline Weight (kg) & $80.25 \pm 15.25$ & $81.32 \pm 15.45$ & $80.79 \pm 15.35$ & 0.144 & 0.892 \\
\hline $\begin{array}{l}\text { BMI } \\
{\left[w t /(h t)^{\wedge} 2\right]}\end{array}$ & $32.78 \pm 6.57$ & $33.78 \pm 6.57$ & $34.78 \pm 6.57$ & 0.016 & 0.760 \\
\hline $\begin{array}{l}\text { Gender } \\
\text { Male } \\
\text { Female }\end{array}$ & $\begin{array}{l}20(66.7 \%) \\
10(33.3 \%)\end{array}$ & $\begin{array}{l}18(60.0 \%) \\
12(40.0 \%)\end{array}$ & $\begin{array}{c}22(73.3 \%) \\
8(26.7 \%)\end{array}$ & $1.202 \#$ & 0.459 \\
\hline $\begin{array}{l}\text { ASA stratus } \\
I \\
I I\end{array}$ & $\begin{array}{c}23(76.7 \%) \\
7(23.3 \%)\end{array}$ & $\begin{array}{c}22(73.3 \%) \\
8(26.7 \%)\end{array}$ & $\begin{array}{c}24(80.0 \%) \\
6(20.0 \%)\end{array}$ & $0.373 \#$ & 0.830 \\
\hline $\begin{array}{l}\text { Type of } \\
\text { surgery } \\
\text { Rhinoplasty } \\
\text { FEES } \\
\text { Turbinectomy }\end{array}$ & $\begin{array}{c}16(53.4 \%) \\
7(23.3 \%) \\
7(23.3 \%)\end{array}$ & $\begin{array}{c}14(46.7 \%) \\
7(23.3 \%) \\
9(30 \%)\end{array}$ & $\begin{array}{c}15(50.0 \%) \\
8(26.7 \%) \\
7(23.3 \%)\end{array}$ & 2.395\# & 0.664 \\
\hline
\end{tabular}

F- One Way Analysis of variance; \#x2: Chi-square test

All data expressed by Mean $\pm S D$

Post HOC: + Significant difference between group $A ;+$ Significant difference between group $B$ p-value $>0.05 \mathrm{NS}$; *p-value <0.05 $\mathrm{S}$; **p-value <0.001 HS

This table shows statistically non-significant (NS) difference between the groups regarding demographic data. 
Table (1): Comparison between the groups regarding intraoperative data.

\begin{tabular}{|l|c|c|c|c|c|}
\hline Intraoperative data & $\begin{array}{c}\text { Group (A): } \\
\text { Standard } \\
(\mathbf{n = 3 0})\end{array}$ & $\begin{array}{c}\text { Group (B): } \\
\text { No } \\
\text { stimulation } \\
(\mathbf{n = 3 0})\end{array}$ & $\begin{array}{c}\text { Group (C): } \\
\text { Dexmedetomidine } \\
(\mathbf{n = 3 0 )}\end{array}$ & F/x2\# & $\begin{array}{c}\text { p- } \\
\text { value }\end{array}$ \\
\hline $\begin{array}{l}\text { Duration of anesthesia } \\
\text { (min) }\end{array}$ & $151.20 \pm 23.98$ & $149.04 \pm 28.73$ & $147.96 \pm 22.68$ & 0.316 & 0.735 \\
\hline Duration of surgery (min) & $128.96 \pm 21.92$ & $126.88 \pm 21.57$ & $125.84 \pm 21.39$ & 0.570 & 0.571 \\
\hline
\end{tabular}

All data expressed by Mean $\pm S D$

p-value >0.05 NS; *p-value $<0.05 \mathrm{~S}$; **p-value <0.001 HS

This table shows statistically (NS) difference between all groups regarding intraoperative data.

Table (2): Comparison between the groups regarding extubation Time (min).

\begin{tabular}{|l|c|c|c|c|c|}
\hline Extubation Time (min) & $\begin{array}{c}\text { Group } \\
\mathbf{( A ) :} \\
\text { Standard } \\
(\mathbf{n}=\mathbf{3 0})\end{array}$ & $\begin{array}{c}\text { Group (B): } \\
\mathbf{N o} \\
\text { stimulation } \\
(\mathbf{n = 3 0})\end{array}$ & $\begin{array}{c}\text { Group (C): } \\
\text { Dexmedetomidine } \\
(\mathbf{n = 3 0})\end{array}$ & ANOVA & p-value \\
\hline Extubation Time (min) & $8.96 \pm 2.81$ & $15.34 \pm 2.59 \%$ & $8.15 \pm 4.35 \%$ & 9.144 & $<0.001 * *$ \\
\hline
\end{tabular}

All data expressed by Mean $\pm S D$

p-value >0.05 NS; *p-value <0.05 S; **p-value <0.001 HSThis table shows statistically significant difference

between the groups regarding extubation time ( $\mathrm{min})$.

Table (3): Comparison between the groups regarding HR (beat/min).

\begin{tabular}{|c|c|c|c|c|c|}
\hline $\begin{array}{l}\text { Heart Rate } \\
\text { (beat/min) }\end{array}$ & $\begin{array}{c}\text { Group (A): } \\
\text { Standard } \\
(\mathrm{n}=\mathbf{3 0})\end{array}$ & $\begin{array}{c}\text { Group (B): } \\
\text { No } \\
\text { Stimulation } \\
(n=30)\end{array}$ & $\begin{array}{c}\text { Group }(\mathbf{C}): \\
\text { Dexmedetomidine } \\
(\mathbf{n}=\mathbf{3 0})\end{array}$ & ANOVA & p-value \\
\hline Baseline & $81.12 \pm 4.06$ & $81.12 \pm 4.06$ & $79.09 \pm 3.95$ & 1.963 & 0.159 \\
\hline At 2 min. & $83.15 \pm 4.16$ & $81.12 \pm 4.06$ & $78.08 \pm 3.90$ & 1.596 & 0.149 \\
\hline At $5 \mathrm{~min}$. & $120.67 \pm 6.03$ & $84.16 \pm 4.21 \%$ & $75.04 \pm 3.75+t$ & 9.706 & $0.002 *$ \\
\hline At $10 \mathrm{~min}$. & $126.75 \pm 6.34$ & $98.36 \pm 4.92 \%$ & $74.02 \pm 3.70+t$ & 6.525 & $0.034^{*}$ \\
\hline At $15 \mathrm{~min}$. & $123.71 \pm 6.19$ & $102.41 \pm 5.12 \dagger$ & $69.97 \pm 3.50+t$ & 12.080 & $<0.001 * *$ \\
\hline At 20min. & $111.54 \pm 5.58$ & $99.37 \pm 4.97 \%$ & $76.05 \pm 3.80 \% t$ & 7.178 & $0.019^{*}$ \\
\hline At $25 \mathrm{~min}$. & $93.29 \pm 4.66$ & $90.25 \pm 4.51$ & $86.16 \pm 4.21$ & 0.525 & 0.468 \\
\hline At 30min. & $83.15 \pm 4.16$ & $84.16 \pm 4.21$ & $81.12 \pm 4.06$ & 0.034 & 0.854 \\
\hline
\end{tabular}

All data expressed by Mean $\pm S D$

p-value >0.05 NS; *p-value <0.05 S; **p-value <0.001 HS

This table shows statistically significant difference between the groups regarding HR at 5, 10, 20 and highly significant at $15 \mathrm{~min}$.

Table (4): Comparison between the groups regarding SBP (mmHg).

\begin{tabular}{|l|c|c|c|c|c|}
\hline $\begin{array}{l}\text { Systolic blood } \\
\text { pressure }(\mathbf{m m H g})\end{array}$ & $\begin{array}{c}\text { Group (A): } \\
\text { Standard } \\
(\mathbf{n = 3 0})\end{array}$ & $\begin{array}{c}\text { Group (B): } \\
\text { No } \\
\text { Stimulation } \\
(\mathbf{n = 3 0 )}\end{array}$ & $\begin{array}{c}\text { Group (C): } \\
\text { Dexmedetomidine } \\
(\mathbf{n = 3 0 )}\end{array}$ & ANOVA & p-value \\
\hline Baseline & $111.54 \pm 5.58$ & $110.53 \pm 5.53$ & $116.61 \pm 5.83$ & 0.525 & 0.468 \\
\hline At 2min. & $114.58 \pm 5.73$ & $110.53 \pm 5.53$ & $112.55 \pm 5.63$ & 0.683 & 0.140 \\
\hline At 5min. & $139.93 \pm 7.00$ & $114.58 \pm 5.73 \dagger$ & $109.51 \pm 5.48+\neq$ & 9.380 & $0.009^{*}$ \\
\hline At 10min. & $141.96 \pm 7.10$ & $121.68 \pm 6.08 \dagger$ & $107.48 \pm 5.37 \dagger \neq$ & 7.527 & $0.023^{*}$ \\
\hline At 15min. & $133.85 \pm 6.69$ & $121.68 \pm 6.08 \dagger$ & $106.47 \pm 5.32 \dagger \neq$ & 8.038 & $0.011^{*}$ \\
\hline At 20min. & $131.82 \pm 6.59$ & $121.68 \pm 6.08 \dagger$ & $114.58 \pm 5.73 \dagger \neq$ & 5.011 & $0.029^{*}$ \\
\hline At 25min. & $124.72 \pm 6.24$ & $120.67 \pm 6.03$ & $119.65 \pm 5.98$ & 0.034 & 0.854 \\
\hline At 30min. & $121.68 \pm 6.08$ & $120.67 \pm 6.03$ & $115.60 \pm 5.78$ & 0.095 & 0.758 \\
\hline
\end{tabular}

All data expressed by Mean $\pm S D$,p-value $>0.05 \mathrm{NS} ; *$ p-value $<0.05 \mathrm{~S} ; * *$-value $<0.001 \mathrm{HS}$

This table shows statistically significant difference between the groups regarding SBP ( $\mathrm{mmHg}$ ) At 5,10,15, and $20 \mathrm{~min}$. 
ejhm.journals.ekb.eg

Table (5): Comparison between the groups regarding DBP (mmHg).

\begin{tabular}{|c|c|c|c|c|c|}
\hline $\begin{array}{l}\text { Diastolic blood } \\
\text { pressure (mmHg) }\end{array}$ & $\begin{array}{c}\text { Group (A): } \\
\text { Standard } \\
(\mathbf{n}=\mathbf{3 0})\end{array}$ & $\begin{array}{c}\text { Group (B): } \\
\text { No } \\
\text { Stimulation } \\
(\mathbf{n}=\mathbf{3 0})\end{array}$ & $\begin{array}{c}\text { Group }(\mathbf{C}): \\
\text { Dexmedetomidine } \\
(\mathrm{n}=30)\end{array}$ & ANOVA & p-value \\
\hline Baseline & $68.95 \pm 3.45$ & $65.91 \pm 3.30$ & $68.95 \pm 3.45$ & 0.065 & 0.967 \\
\hline At $2 \mathrm{~min}$. & $70.98 \pm 3.55$ & $67.94 \pm 3.40$ & $66.92 \pm 3.35$ & 0.260 & 0.609 \\
\hline At $5 \mathrm{~min}$. & $93.29 \pm 4.66$ & $71.99 \pm 3.60 \%$ & $62.87 \pm 3.14 t+$ & 6.585 & $0.037 *$ \\
\hline At $10 \mathrm{~min}$. & $96.33 \pm 4.82$ & $81.12 \pm 4.06 \%$ & $65.91 \pm 3.30 \% t$ & 8.696 & $0.013^{*}$ \\
\hline At $15 \mathrm{~min}$. & $93.29 \pm 4.66$ & $85.18 \pm 4.26 \dagger$ & $66.92 \pm 3.35+t$ & 11.040 & $<0.001 * *$ \\
\hline At $20 \mathrm{~min}$. & $90.25 \pm 4.51$ & $83.15 \pm 4.16 \dagger$ & $73.01 \pm 3.65+t$ & 8.598 & $0.014 *$ \\
\hline At $25 \mathrm{~min}$. & $81.12 \pm 4.06$ & $79.09 \pm 3.95$ & $76.05 \pm 3.80$ & 0.095 & 0.758 \\
\hline At $30 \mathrm{~min}$. & $76.05 \pm 3.80$ & $74.02 \pm 3.70$ & $73.01 \pm 3.65$ & 1.067 & 0.587 \\
\hline
\end{tabular}

All data expressed by Mean $\pm S D$

p-value >0.05 NS; *p-value <0.05 S;**p-value <0.001 HS

This table shows statistically significant difference between the groups regarding DBP at 5, 10,15 and $20 \mathrm{~min}$.

Table (6): Comparison between the groups regarding coughing.

\begin{tabular}{|c|c|c|c|c|c|}
\hline Coughing & $\begin{array}{c}\text { Group (A): } \\
\text { Standard } \\
(n=30)\end{array}$ & $\begin{array}{c}\text { Group (B): } \\
\text { No } \\
\text { Stimulation } \\
(n=30)\end{array}$ & $\begin{array}{c}\text { Group }(C): \\
\text { Dexmedetomidine } \\
(n=30)\end{array}$ & $\begin{array}{c}\text { Chi- } \\
\text { square } \\
\text { test }\end{array}$ & p-value \\
\hline Grade 0 & $0(0.0 \%)$ & $3(10.0 \%)$ & $21(70 \%)+t$ & 43.977 & $<0.001 * *$ \\
\hline Grade 1 & $4(13.3 \%)$ & $22(73.3 \%)+$ & $6(20 \%) \ddagger$ & 10.201 & $0.006^{*}$ \\
\hline Grade 2 & $20(66.7 \%)$ & $5(16.7 \%)+$ & $3(10 \%)+$ & 26.855 & $<0.001 * *$ \\
\hline Grade 3 & $6(20.0 \%)$ & $0(0.0 \%)+$ & $0(0 \%)+$ & 12.857 & $0.002 *$ \\
\hline Grade 4 & $0(0.0 \%)$ & $0(0.0 \%)$ & $0(0 \%)$ & 0.000 & 1.000 \\
\hline
\end{tabular}

p-value >0.05 NS; *p-value <0.05 S; **p-value <0.001 HS

This table shows statistically significant difference between the groups regarding coughing Grade 1, 3 and highly significant at grade 0,2 .

Table (7): Comparison between the groups regarding laryngospasm.

\begin{tabular}{|l|c|c|c|c|c|}
\hline Laryngospasm & $\begin{array}{c}\text { Group (A): } \\
\text { Standard } \\
(\mathbf{n = 3 0})\end{array}$ & $\begin{array}{c}\text { Group (B): } \\
\text { No } \\
\text { Stimulation } \\
(\mathbf{n = 3 0 )}\end{array}$ & $\begin{array}{c}\text { Group (C): } \\
\text { Dexmedetomidine } \\
(\mathbf{n = 3 0 )}\end{array}$ & $\begin{array}{c}\text { Chi- } \\
\text { square } \\
\text { test }\end{array}$ & p-value \\
\hline Grade 0 & $24(80.0 \%)$ & $29(96.7 \%) \dagger$ & $30(100 \%) \dagger$ & 9.604 & $0.008^{*}$ \\
\hline Grade 1 & $4(13.3 \%)$ & $1(3.3 \%) \dagger$ & $0(0 \%) \dagger$ & 6.506 & $0.044^{*}$ \\
\hline Grade 2 & $2(6.7 \%)$ & $0(0.0 \%)$ & $0(0 \%)$ & 4.725 & 0.094 \\
\hline Grade 3 & $0(0.0 \%)$ & $0(0.0 \%)$ & $0(0 \%)$ & 0.000 & 1.000 \\
\hline
\end{tabular}

p-value $>0.05 \mathrm{NS} ;{ }^{*}$ p-value $<0.05 \mathrm{~S}$

This table shows statistically significant difference between the groups regarding laryngospasm Grade 0 and grade 1 .

Table (8): Comparison between the groups regarding oozing from the wound.

\begin{tabular}{|l|c|c|c|c|c|}
\hline $\begin{array}{l}\text { Oozing from the } \\
\text { wound }\end{array}$ & $\begin{array}{c}\text { Group (A): } \\
\text { Standard } \\
(\mathbf{n}=\mathbf{3 0})\end{array}$ & $\begin{array}{c}\text { Group (B): } \\
\text { No } \\
\text { Stimulation } \\
(\mathbf{n = 3 0 )}\end{array}$ & $\begin{array}{c}\text { Group (C): } \\
\text { Dexmedetomidine } \\
(\mathbf{n}=\mathbf{3 0})\end{array}$ & $\begin{array}{c}\text { Chi- } \\
\text { square } \\
\text { test }\end{array}$ & p-value \\
\hline Grade 0 & $0(0.0 \%)$ & $0(0.0 \%)$ & $0(0 \%)$ & 0.000 & 1.000 \\
\hline Grade 1 & $7(23.3 \%)$ & $21(70.0 \%) \dagger$ & $25(83.3 \%) \dagger$ & 24.600 & $<0.001^{* *}$ \\
\hline Grade 2 & $15(50.0 \%)$ & $6(20.0 \%) \dagger$ & $3(10 \%) \dagger$ & 13.295 & $0.003^{*}$ \\
\hline Grade 3 & $8(26.7 \%)$ & $3(10.0 \%) \dagger$ & $2(6.7 \%) \dagger$ & 5.574 & $0.041^{*}$ \\
\hline
\end{tabular}

p-value >0.05 NS; *p-value <0.05 S; **p-value <0.001 HS

This table shows statistically significant difference between the groups regarding oozing from the wound Grade 1 ,

2 and grade 3. 
Table (9): Comparison between the groups regarding airway events-related complications.

\begin{tabular}{|l|c|c|c|c|c|}
\hline $\begin{array}{l}\text { Airway Events-related } \\
\text { Complications }\end{array}$ & $\begin{array}{c}\text { Group (A): } \\
\text { Standard } \\
(\mathbf{n = 3 0})\end{array}$ & $\begin{array}{c}\text { Group (B): } \\
\text { No } \\
\text { stimulation } \\
(\mathbf{n = 3 0 )}\end{array}$ & $\begin{array}{c}\text { Group (C): } \\
\text { Dexmedetomidine } \\
(\mathbf{n = 3 0 )}\end{array}$ & $\begin{array}{c}\text { Chi- } \\
\text { square } \\
\text { test }\end{array}$ & p-value \\
\hline Excessive secretion & $14(46.7 \%)$ & $5(16.7 \%) \dagger$ & $4(13.3 \%) \dagger$ & 10.629 & $0.005^{*}$ \\
\hline Breath holding & $13(43.3 \%)$ & $3(10.0 \%) \dagger$ & $0(0 \%) \dagger$ & 21.132 & $<0.001^{* *}$ \\
\hline $\begin{array}{l}\text { Sever desaturation } \\
\text { Episodes (spo2<90\%) }\end{array}$ & $4(13.3 \%)$ & $0(0.0 \%) \dagger$ & $0(0 \%) \dagger$ & 8.372 & $0.015^{*}$ \\
\hline Emergence agitation & $17(56.7 \%)$ & $5(16.7 \%) \dagger$ & $1(3.35 \%) \dagger$ & 24.296 & $<0.001^{* *}$ \\
\hline Biting & $10(33.3 \%)$ & $4(13.3 \%) \dagger$ & $3(10.0 \%) \dagger$ & 6.237 & $0.044^{*}$ \\
\hline Hoarseness & $6(20.0 \%)$ & $0(0.0 \%) \dagger$ & $0(0 \%) \dagger$ & 12.857 & $0.002^{*}$ \\
\hline
\end{tabular}

p-value >0.05 NS; *p-value <0.05 S; **p-value <0.001 HS

This table shows statistically significant difference between the different groups regarding airway events related complications.

\section{DISCUSSION}

As known during emergence extubation is associated with stimulation of the sympathetic adrenal system and the release of catecholamines which is the possible mechanism responsible for hypertension, tachycardia and arrythmeia after tracheal extubation ${ }^{(\mathbf{1 4})}$.

As regard circulatory reflexes the present results showed that the changes in HR, SBP, and DBP during emergence extubation were significantly less in "no stimulation" technique and dexmedetomidine groups than group A. This could be explained as removal of the TT by "no stimulation " technique was more effective than standard awake extubation in controlling circulatory reflexes and this results may be attributed to the inhibition of catecholamine release which was a direct result of not disturbing the patients' tracheas, less airwayrelated events, and smoother emergence. These results were coincided with Sheta $\boldsymbol{e t}$ al. ${ }^{(15)}$ where dexmedetomidine is a potent alpha2 adrenoceptor agonist, that decrease the sympathetic outflow and noradrenergic activity thereby counteracting the hemodynamic fluctuation occurring at the time of extubation. Also, dexmedetomidine activates receptors in the medullary vasomotor center, reducing norepinephrine turnover and decreasing central sympathetic outflow ${ }^{(\mathbf{1 6})}$.

Recovering from anesthesia often results in elevated catecholamine concentration following anesthetic withdrawal which is further aggravated by laryngeal manipulation occurring during extubation.

There were significantly lower increases in HR, SBP, and DBP after extubation in patients who received dexmedetomidine compared with groups $\mathrm{A}$ and B and this was coincided with Kothari et $\boldsymbol{a l} .{ }^{(7)}$ who found that a single dose of dexmedetomidine 0.5 $\mu \mathrm{g} / \mathrm{kg}$ given $5 \mathrm{~min}$ before extubation produced better attenuation of hemodynamic response in craniotomy patients. However, Sharma et al. ${ }^{(\mathbf{(})}$ found decrease in mean arterial pressure after $5 \mathrm{~min}$ from baseline value.
Our results were coincided with Guler $\boldsymbol{e t}$ al . (11) who reported that dexmedetomidine $0.5 \mu \mathrm{g} / \mathrm{kg}$ was associated with attenuation of circulatory reflexes on emergence from anesthesia in intraocular surgery, without prolonged recovery. HR, SBP, and DBP were significantly increased from baseline at extubation in both groups $(\mathrm{P}<0.05)$, but the increase was significantly less substantial with dexmedetomidine.

Our results were also coincided with Talke et al. (17) who compared the effects of dexmedetomidine with isotonic saline on hemodynamic changes in patients who had undergone vascular surgery. That study found that the dexmedetomidine I.V infusion was associated with significant attenuation of increases in HR, BP, and plasma catecholamine concentrations during emergence from anesthesia compared with placebo $(\mathrm{P}<0.05)$.

Regarding airway-related complications (coughing, bucking, laryngospasm and oozing from the wound) the results of our study showed that the incidence of coughing and bucking were significantly less in group B and C more than group A which can be explained as the TT and its cuff stimulate the rapidly acting irritant and the stretch receptors in the trachea ${ }^{(\mathbf{1 8})}$. Those irritant receptors are assumed to be the receptors involved in the cough reflex ${ }^{(19)}$. The blockade of these receptors is the theoretical reason behind inhibition of the cough reflex during extubation by topical application of local anesthetics (20).

The same concept can be applied to the "notouch" technique. There, we avoided excessive tracheal irritation by early deflation of the cuff of the endotracheal tube. Similarly, any stimulation such as oropharyngeal suctioning, head turning, pillow removal, and bodily movements that causes tracheal irritation and awakening in our patients were not allowed. Therefore, severity of emergence-related coughing in patients extubated with this technique was significantly less. 
In dexmedetomidine group $0.5 \mu \mathrm{g} / \mathrm{kg}$ IV before tracheal extubation was associated with significantly less coughing and better quality of extubation than group $\mathrm{A}$ and $\mathrm{B}$ because dexmedetomidine, is a potent alpha2 adrenoceptor agonist, decrease the sympathetic outflow and noradrenergic activity thereby and possessed both sedated and analgesic activity ${ }^{(\mathbf{1 6})}$.

Our results were coincided with Guler $\boldsymbol{e t} \boldsymbol{a l}$. (11) who reported that dexmedetomidine $0.5 \mu \mathrm{g} / \mathrm{kg}$ was associated with attenuation of the prevalence and severity of airway-related complications on emergence from anesthesia in intraocular surgery, without prolonged recovery. Cough scores were significantly lower in the dexmedetomidine group than in the placebo group $(\mathrm{P}<0.05)$, but there were no differences between-group in the prevalence of breath holding and desaturation.

Our results were also, coincided with Aksu et al. (9) who did a similar study comparing fentanyl with dexmedetomidine. The fentanyl group had a similar prevalence of cough (70\%), 20\% of which was severe. The lower prevalence of cough (15\%) found with dexmedetomidine compared with fentanyl suggests that dexmedetomidine was more effective for improving the quality of extubation.

Fan et $\boldsymbol{a l l}^{(\mathbf{( 2 1 )}}$ who did a similar study comparing remifentanil with two doses of dexmedetomidine $0.5 \mu \mathrm{g} / \mathrm{kg}$ and $0.7 \mu \mathrm{g} / \mathrm{kg}$ for otology surgery and observed that higher percentage of patients in dexmedetomidine group had smooth extubation regarding the absence of bucking and coughing with head movement during surgical dressing, they also observed the incidence of postoperative nausea and vomiting was less with dexmedetomidine group.

As regard laryngeal spasm, both groups B and $\mathrm{C}$ in the present study, showed no case of laryngeal spasm or severe desaturation episodes. In contrary, there were 3 cases of laryngeal spasm in patients who had their trachea extubated with standard awake technique. Less airway stimulation and smooth recovery adopted by "non touch" technique and dexmedetomidine can again explain the lower incidence of laryngeal spasm. Another explanation is lesser oozing from the wound. Bleeding from surgical sites and leakage of blood through postnasal space can contaminate the vocal folds, potentially giving rise to laryngeal spasm and these results were coincided with that obtained by Sheta et al. ${ }^{(15)}$ and Aksu et al. ${ }^{(9)}$.

As regard vomiting, our results showed that four patients (two in each group A and B) vomited in the first 30 minutes after extubation, all were awake and they were able to protect their own airway, with no evidence of aspiration while there is no case had vomiting in group $\mathrm{C}$. This emphasizes the importance of delaying extubation until patients are sufficiently awake to voluntarily protect their airways and these results were coincided with the results of Sheta $\boldsymbol{e t}$ al. (15).

As known as emergence agitation is a concern problem during extubation in nasal and paranasal surgeries, our results showed that emergence agitation in group A was more than group $\mathrm{B}$ and $\mathrm{C}$ results which coincided with Epstein SK. who found that the dexmedetomidine reduce the prevalence of emergence agitation ${ }^{(22)}$.

Regarding extubation time (from the time of sevoflurane closure until extubation), it was prolonged in group B more than groups A and C.

Our results showed that extubation time was prolonged in group B more than group A and C. Prolonged duration of emergence from GA encountered in the "no stimulation" technique may be considered as a drawback. However, when it comes to patient safety, it is probably a minor concern. As shown in the present study, the technique produces fewer airway-related complications, as well as minimal hemodynamic response, and most importantly is the absence of laryngeal spasm or severe desaturation episodes. Alternatively, as compared with deep tracheal extubation, the latter delay emergence time even more ${ }^{(23)}$, and unfavorably prolongs the time from tracheal extubation to return of protective airway reflexes.

In dexmedetomidine group extubation time was shorter than group $\mathrm{A}$ and $\mathrm{B}$ and this was explained as the distribution phase of dexmedetomidine is rapid, with a half-life of distribution of approximately 6 minutes and elimination half-life of 2 hours. The average protein binding is $94 \%$ and is constant across the different plasma concentrations and similar in males and females ${ }^{(24)}$.

Therefore, this technique (dexmedetomidine) is a safe alternative for tracheal extubation and it may be favored as extubation technique of choice, particularly in airway surgery or when the patient or surgical risk factors require that coughing and hypertension during emergence from anesthesia is prohibited.

As regard sore throat although the severity of coughing in the "no stimulation" and dexmedetomidine groups was low, it did not result in a significant lower incidence of sore throat, in comparison with standard awake group.

Postoperative sore throat may relate not only to the anesthesia technique, but also to patients' factors and type of surgical procedures ${ }^{(25)}$. Besides laryngoscopy and endotracheal intubation, in certain surgical procedures. Sore throat can occur as a consequence of localized trauma secondary to surgery itself, throat packing, and excessive oral suction $^{(26)}$. 


\section{CONCLUSION}

From the previous results we can conclude that the dexmedetomidine group was associated with minimal circulatory reflexes and airway-related complications without prolonged recovery compared with the "no stimulation" extubation technique.

\section{REFERENCES}

1. Minogue SC, Ralph J, Lampa MJ (2004): Laryngotracheal topicalization with lidocaine before intubation decreases the incidence of coughing on emergence from general anesthesia. Anesth Analg., 99:1253-7.

2. Mistry T, Purohit S, Arora G, Gill N, Sharma J (2016): Attenuation of extubation responses: comparison of prior treatment with verapamil and dexmedetomidine. Journal of Neuro anesthesiology and Critical Care, 3(01):033-9.

3. Luthra A, Prabhakar H, Rath GP (2017): Alleviating stress response to tracheal extubation in neurosurgical patients: a comparative study of two infusion doses of dexmedetomidine. Journal of Neurosciences in Rural Practice, 8(1):49-56.

4. Patel HP, Shashank MR, Shivaramu BT (2018): Attenuation of hemodynamic response to tracheal extubation: A comparative study between esmolol and labetalol. Anesthesia, Essays and Researches, 12 (1):180-188.

5. Lim SH, Chin NM, Tai HY, Wong M, Lin TK (2000): Prophylactic esmolol infusion for the control of cardiovascular responses to extubation after intracranial surgery. Ann Acad Med Singapore, 29:447-51.

6. Jee D, Park SY (2003): Lidocaine sprayed down the endotracheal tube attenuates the airway-circulatory reflexes by local anesthesia during emergence and extubation. Anesth Analg., 96:293-7.

7. Kothari D, Tandon N, Singh M, Kumar A (2014): Attenuation of circulatory and airway responses to endotracheal extubation in craniotomies for intracerebral space occupying lesions: Dexmedetomidine versus lignocaine. Anesth Essays Res., 8:78-82.

8. Sharma VB, Prabhakar H, Rath GP, Bithal PK (2014): Comparison of dexmedetomidine and lignocaine on attenuation of airway and pressor responses during tracheal extubation. J Neuroanaesth Crit Care, 1:50-5.

9. Aksu R, Akin A, Biçer C, Esmaoglu A, Tosun Z, Boyaci A (2009): Comparison of the effects of dexmedetomidine versus fentanyl on airway reflexes and hemodynamic responses to tracheal extubation during rhinoplasty: A double-blind, randomized, controlled study. Curr Ther Res Clin Exp., 70:209-20.

10. Kim ES, Bishop MJ (1998): Cough during emergence from isoflurane anesthesia. Anesth Analg., 87:1170-4.

11. Guler G, Akin A, Tosun Z, Eskitascoglu E, Mizrak A, Boyaci A (2005): Single dose dexmedetomidine attenuates airway and circulatory reflexes during extubation. Acta Anaesthesiol Scand., 49:1088-91.

12. Tsui BC, Wagner A, Cave D, Elliott C, El-Hakim H, Malherbe S (2004): The incidence of laryngospasm with a "No Touch" extubation technique after tonsillectomy and adenoidectomy. Anesth Analg., 98:327-9.

13. Rani P, Hemanth Kumar VR, Ravishankar M, Sivashanmugam T, Sripriya R, Trilogasundary $M$ (2016): Rapid and reliable smooth extubation Comparison of fentanyl with dexmedetomidine: A randomized, double-blind clinical trial. Anesth Essays Res., 10:597-601.

14. Jain V, Chaturvedi A, Pandia MP, Bithal PK (2019): Effect of dexmedetomidine on recovery profile of patients undergoing anterior cervical discectomy and fusion. Journal of Anaesthesiology, Clinical Pharmacology, 35(1):92-69.

15. Sheta SA, Abdelhalim AA, Nada E (2011): Evaluation of "no touch" extubation technique on airway-related complications during emergence from general anesthesia. Saudi Journal of Anaethesia, 5(2):125-31.

16. Jain D, Khan R, Maroof $M$ (2008): Effect of dexmeditomidine on stress response to extubation. Internet J Anaesthesiol., 21:21-2.

17. Talke P, Chen R, Thomas B et al. (2000): The hemodynamic and adrenergic effects of perioperative dexmedetomidine infusion after vascular surgery. Anesth Analg., 90:834-839.

18. Canning BJ (2006): Anatomy and neurophysiology of the cough reflex: ACCP evidence-based clinical practice guidelines. Chest, 129:33S-47.

19. Safavi M, Honarmand A, Khazaei M (2016): The effects of propofol, ketamine and combination of them in prevention of coughing and laryngospasm in patients awakening from general anesthesia: a randomized, placebo-controlled, double blind clinical trial. Advanced Biomedical Research, 5:64.

20. Diachun CA, Tunink BP, Brock-Utne JG (2001): Suppression of cough during emergence from general anesthesia: Laryngotracheal lidocaine through a modified endotracheal tube. J Clin Anesth., 13:447-51.

21. Fan Q, Hu C, Ye M, Shen X (2015): Dexmedetomidine for tracheal extubation in deeply anesthetized adult patients after otologic surgery: A comparison with remifentanil. BMC Anesthesiol., 15:106-10.

22. Epstein SK (2002): Decision to extubate. Intensive Care Med., 28(5):535-546.

23. Begum U, Singh PR, Naithani B, Singh V, Singh GP, Tiwari T (2019): Dexmedetomidine as bolus or lowdose infusion for the prevention of emergence agitation with sevoflurane anesthesia in pediatric patients. Anesthesia, Essays and Researches, 13(1):57.

24. Gertler R, Brown HC, Mitchell DH, Silvius EN (2001): Dexmedetomidine: A novel sedative-analgesic agent. Proc (Bayl Univ Med Cent), 14:13-21.

25. Higgins PP, Chung F, Mezei G (2002): Postoperative sore throat after ambulatory surgery. $\mathrm{Br} \mathrm{J}$ Anaesth., 88:582-4.

26. Agarwal A, Nath SS, Goswami D, Gupta D, Dhiraaj S, Singh PK (2006): An evaluation of the efficacy of aspirin and benzydamine hydrochloride gargle for attenuating postoperative sore throat: A prospective randomized, single- blind study. Anesth Analg., 103:1001-3. 